\title{
Shape memory effect in nanoindentation of nickel-titanium thin films
}

\author{
G. A. Shaw \\ Department of Chemistry, University of Wisconsin-Madison, Madison, Wisconsin 53706 \\ D. S. Stone \\ Materials Science Department, University of Wisconsin-Madison, Madison, Wisconsin 53706
}

A. D. Johnson

TiNi Alloy Company, San Leandro, California 94577

A. B. Ellis

Department of Chemistry, University of Wisconsin-Madison, Madison, Wisconsin 53706

W. C. Crone ${ }^{\text {a) }}$

Department of Engineering Physics, University of Wisconsin-Madison, Madison, Wisconsin 53706

(Received 23 October 2002; accepted 12 May 2003)

In this study, a series of nanoindentations was made on NiTi shape memory alloy thin films at millinewton loads with a Berkovich indenter. Mapping of the indentation topography using atomic force microscopy reveals direct evidence that the thermally induced martensitic transformation of these films allows for partial indent recovery on the nanoscale. Indeed, recovery is nearly complete at indentation depths of less than $100 \mathrm{~nm}$. A hemispherical cavity model is presented to predict an upper limit to shape memory recovery of sharp indentations. (C) 2003 American Institute of Physics. [DOI: $10.1063 / 1.1591235]$

The shape memory effect (SME) is a visually striking phenomenon whereby a material is able to recover its initial shape after significant deformation through subsequent heating. In the case of shape memory alloys (SMAs), the effect arises from a thermally induced atomic level structural change. This solid-state phase change is known as a martensitic transformation and occurs in a wide variety of metal alloys, including the well-known material nitinol (NiTi). ${ }^{1}$

In its low temperature (martensite) phase, the NiTi unit cell is monoclinic, but it changes to a $\mathrm{CsCl}$ (austenite) form upon heating past the phase transformation temperature. In martensitic form, the material can be easily deformed through twin rearrangement. Shape recovery occurs when the material is heated, transforming the martensite to austenite. Due to these remarkable mechanical properties, exceptional robustness, and good biocompatibility, ${ }^{2} \mathrm{NiTi}$ is the most widely used of the SMAs. Because it has the highest known work density of any actuation scheme $\left(\sim 10^{7} \mathrm{~J} / \mathrm{m}^{3}\right),^{3}$ and is amenable to thin film deposition processes, ${ }^{4,5} \mathrm{NiTi}$ is also an attractive candidate for use in micro- and nanoscale actuation.

An area of recent research interest has been the indentation behavior of SMAs. ${ }^{6-13}$ Wangyang et al. have shown that thermally activated martensitic transformations allow recovery of microscale spherical or Vickers indentations in bulk NiTi. ${ }^{13}$ We demonstrate in this study that the same effects occur in thin films of NiTi, and that they can be observed on the nanoscale.

Thin films of NiTi were deposited on oxidized Si by DC magnetron sputtering at TiNi Alloy Co. ${ }^{4}$ Grazing angle incidence $\mathrm{x}$-ray crystallography at room temperature showed the

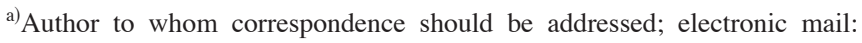
crone@engr.wisc.edu
}

films to be martensite. To probe the SME at the nanoscale, a Hysitron Triboscope Nanoindenter was used in conjunction with a Digital Instruments nanoscope IV atomic force microscope (AFM). The nanoindenter was equipped with a Berkovich tip and attached to the AFM scanner tube, allowing for in situ topographical imaging at low loads. This enabled us to preselect a good location for an indent, avoiding any obvious large topographical excursions, and position the nanoindenter to within a few nanometers of this area. Indentation was then performed at $8,3,1$, and $0.5 \mathrm{mN}$ loads on NiTi thin film samples of thickness 10 or $1.7 \mu \mathrm{m}$.

After indentation, a conventional AFM with a standard cantilever tip was used to map the remnant impression. Subsequently, the films were heated to roughly $200^{\circ} \mathrm{C}$ with a heat gun for $30 \mathrm{~s}$ (the austenite finish temperature, $A_{f}$, is $70^{\circ} \mathrm{C}$, as measured by differential scanning calorimetry). This transformed the martensite to austenite and allowed the SME to occur, as evidenced by a change in the appearance of the films' surfaces: in martensite form, the films appeared macroscopically cloudy, due to buckling of their surfaces caused by stresses introduced after annealing. When they transformed to austenite, the cloudiness vanished as the films flattened. After cooling, the films again became cloudy, indicating a return to the martensite phase, and the indents were rescanned. The topography of the indents before and after heating was analyzed using Digital Instruments software, but the topographical data used for analyses were not flattened.

Figure 1 shows an indentation before and after heating. The profile of each indent was measured from the long end of the characteristic triangular Berkovich indent through its deepest point to provide a uniform measurement of indentation depth. The indentation evidently shrinks significantly as a result of the heating. It appears that the process of mapping indent topography by scanning the Berkovich tip across the 

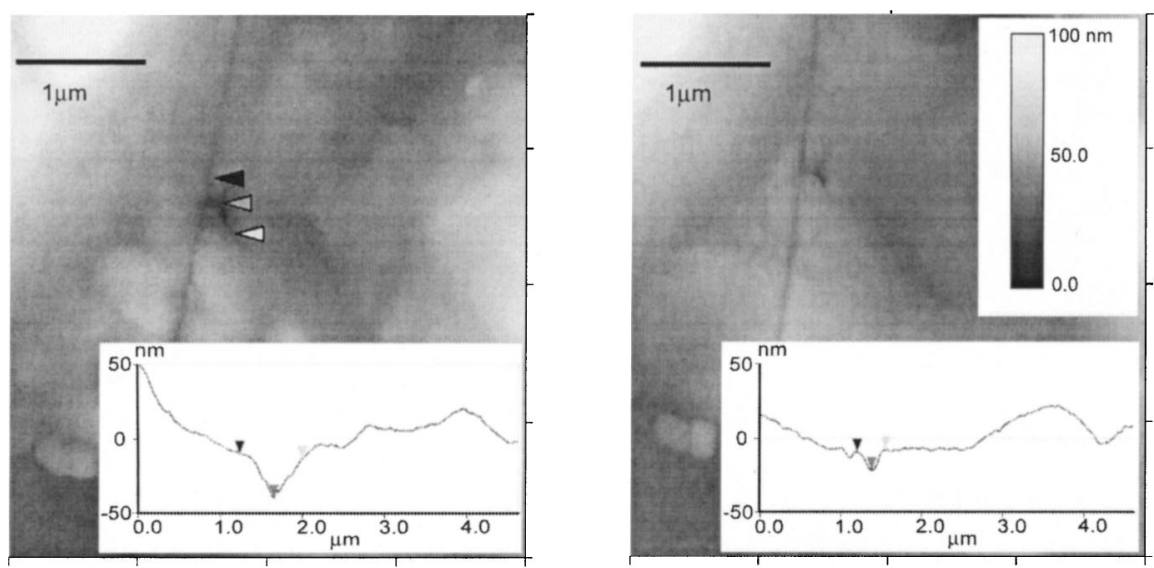

FIG. 1. AFM images of $0.5 \mathrm{mN}$ indentations on a NiTi thin film (left) before and (right) after heating. Insets show indentation depth profiles. The arrows on the profile and AFM image show the points used to calculate $D_{\mathrm{bh}}$ from the left image for this indent; a similar analysis gave $D_{\text {ah }}$ from the other image. sample surface plows small furrows in the film. After heating, this damage repairs itself. Features that were clearly not NiTi (such as small particulates adhering to the surface) had the same dimensions in both images, leading us to conclude that the observed changes in topography are a result of the martensitic transformation rather than a change in experimental conditions.

Examination of the profiles shows that the smaller indents recover a much greater fraction of their depth than the larger ones upon heating. This differs from previous results that showed the recovery from a (Berkovich-like) Vickers indent remains roughly constant, regardless of load, on the microscale. ${ }^{13}$ Figure 2 shows the results for NiTi with spherical and Vickers microindentations compared to the nanoindentations of this study. The recovery ratio is defined as

$$
\text { recovery ratio }=\frac{D_{\mathrm{bh}}-D_{\mathrm{ah}}}{D_{\mathrm{bh}}},
$$

where $D_{\text {bh }}$ and $D_{\text {ah }}$ are maximum indent depth before and after heating, respectively, as measured in Fig. 1.

The higher load data agree well with the previous work, showing that the Berkovich and Vickers indenters behave quite similarly until depths of approximately $100 \mathrm{~nm}$. The fact that the recovery ratio increases for small indents can be interpreted as being due to the spherical end of the Berkovitch indenter tip. The tip's radius of curvature was determined to be approximately $100 \mathrm{~nm}$ using a blind reconstruction technique. ${ }^{14}$ For indents shallower than this depth, the recovery ratio increases as the indent becomes more spherical in character, producing a more uniform stress distribution in the underlying material than would the sharp edges of the

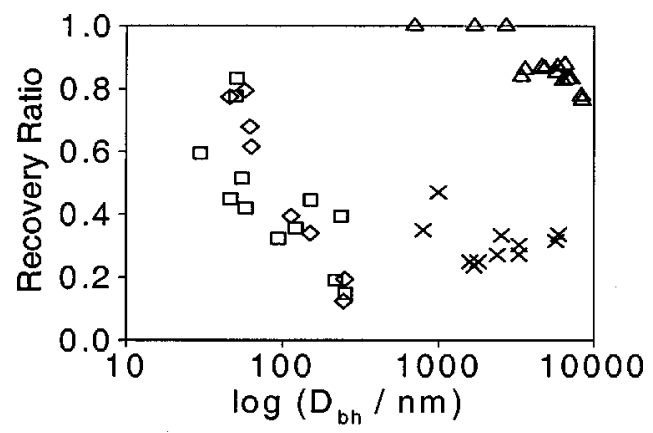

FIG. 2. Comparison of nanoindentation results for $1.7(\square)$ and $10 \mu \mathrm{m}(\diamond)$ thin films to spherical $(\triangle)$ and Vickers $(X)$ microindentation results of $\mathrm{Ni}$ et al. (see Ref. 13).
Berkovich tip geometry. Because the grain size is large ( $\sim 1 \mu \mathrm{m}$ in diameter, as determined by electron backscatter diffraction) compared to the indent size at these depths, the local conditions of the indent area strongly affect its properties. Indentation, tensile and compression testing of single crystal samples ${ }^{8}$ have shown that the mechanical properties of NiTi shape memory alloys depend on crystal orientation. We attribute the scatter at shallow depths to the fact that the smaller indents sample the mechanical properties of single grains which can have differing crystal orientations or grain boundary properties.

The elastic half space illustrated in Fig. 3 has been used to model the indentation process. When the indenter initially contacts the surface, the contact is spherical in nature as a result of the bluntness of the indenter tip. In this low stress state, deformation under the tip is elastic. As indentation depth increases, martensite twin rearrangement is induced, and the Berkovich geometry produces localized high stress regions within the material. Further indentation results in yield when the martensitic rearrangement is incapable of accommodating additional deformation. At this depth, all three processes occur in sequence, as shown in Fig. 3. The radius

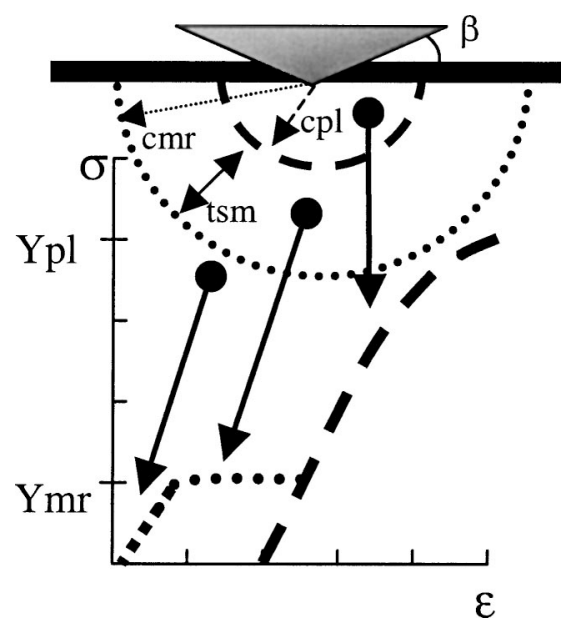

FIG. 3. Elastic half-space diagram of indentation of SMA with comparison to a representative tensile loading curve for martensitic NiTi. In the region farthest from the point of application of the load, the lowest stress field exists, and reversible elastic deformation occurs. Closer to the point, a slightly higher stress field exists where strain is accommodated by martensite twin reorientation. This is the shape memory region that recovers after heating. Finally, at the highest stress state, in direct proximity to the indenter, plastic deformation occurs. 
of the hemisphere that delineates the boundary between elastic deformation and martensitic reorientation is denoted $c_{\mathrm{mr}}$, whereas $c_{\mathrm{pl}}$ delineates the boundary between martensitic reorientation and plastic deformation. Between these hemispheres is a shell of thickness $t_{\mathrm{sm}}$ in which strain has been accommodated through martensite rearrangement; in this region the SME allows shape recovery upon heating.

The case of elastic-perfectly plastic indentation can be examined using the methods reported by Johnson. ${ }^{15}$ For this case, an expression can be derived to locate the elasticplastic boundary during indentation with a hard cone

$$
c=\frac{d}{\tan \beta}\left[\frac{E \tan \beta}{6 Y(1-\nu)}+\frac{2-4 \nu}{3-3 \nu}\right]^{1 / 3} .
$$

Here, $c$ is the radius of the hemisphere delineating the elastic-plastic boundary, $\beta$ is the angle between the surface and the indenter, $E$ is Young's modulus, $\nu$ is Poisson's ratio, and $d$ is indentation depth.

In the present analysis, the loading of NiTi was treated as two separate indentation processes, both of which we took to be well approximated by the elastic-perfectly plastic model. In one process, deformation occurs though martensitic rearrangement at a stress $Y_{\mathrm{mr}}$, and in the other process, it occurs through plastic yielding at a stress $Y_{\mathrm{pl}}$. Using Eq. (2), $c_{\mathrm{mr}}$ and $c_{\mathrm{pl}}$ can be calculated as a function of indentation depth using $Y_{\mathrm{mr}}$ and $Y_{\mathrm{pl}}$, respectively. The difference between these radii gives $t_{\mathrm{sm}}$. Although we could not directly measure $c$, we measured the recovery ratio of the indent after heating. This can be compared to a theoretical recovery ratio, $R_{\mathrm{th}}=t_{\mathrm{sm}} / c_{\mathrm{mr}}=\left(c_{\mathrm{mr}}-c_{\mathrm{pl}}\right) / c_{\mathrm{mr}}$. Assuming the elastic and martensitic rearrangement region have the same $\beta, E$, and $\nu$ values

$$
R_{\mathrm{th}}=1-\left(\frac{Y_{\mathrm{mr}}}{Y_{\mathrm{pl}}}\right)^{1 / 3} \text {. }
$$

For $Y_{\mathrm{mr}}=0.2 \mathrm{GPa}$ and $Y_{\mathrm{pl}}=0.8 \mathrm{GPa}$ (the ultimate tensile yield point of nitinol), ${ }^{1} R_{\text {th }}$ is calculated to be 0.4 , and is constant with indentation depth. The ultimate tensile yield point was chosen as a good estimate of $Y_{\mathrm{pl}}$ despite the fact that yielding can occur at stresses as low as $Y_{\mathrm{mr}}$. In this respect, the $R_{\text {th }}$ value gives a range within which the recov- ery ratio can be predicted. The lower bound, when $Y_{\mathrm{pl}}$ $=Y_{\mathrm{mr}}$, is zero; and the upper bound, when $Y_{\mathrm{pl}}=0.8 \mathrm{GPa}$, is 0.4 . This prediction correlates well with published experimental results ${ }^{13}$ as well as our own experimental results for indentations deeper than $100 \mathrm{~nm}$.

To summarize, nanoindentation AFM was used to characterize the shape memory effect in martensitically transforming NiTi thin films. It was found that a significant fraction of the deformation from the indentation process could be recovered through the shape memory effect upon heating. Johnson's spherical cavity model was used to predict a theoretical recovery ratio of 0.4 which agrees well with indentation data at higher loads. At indentation depths less than about $100 \mathrm{~nm}$, however, a much greater fraction of the deformation was recovered, arguably as a result of the spherical shape of the Berkovich tip at these indentation depths.

The authors wish to thank Professor John Perepezko and Professor Robert Carpick at UW-Madison for valuable discussion and AFM expertise and the Department of Energy for funding.

${ }^{1}$ K. Otsuka and C. M. Wayman, Shape Memory Materials (Cambridge University Press, Cambridge, 1999).

${ }^{2}$ T. Duerig, A. Pelton, and D. Stöckel, Mater. Sci. Eng., A 273-275, 149 (1999).

${ }^{3}$ P. Krulevitch, A. P. Lee, P. V. Ramsey, J. C. Trevino, J. Hamilton, and M. A. Northrup, J. Microelectromech. Syst. 5, 270 (1996).

${ }^{4}$ J. D. Busch, A. D. Johnson, C. H. Lee, and D. A. Stevenson, J. Appl. Phys. 68, 6224 (1990)

${ }^{5}$ L. Chang, C. Hu. Simpson, D. S. Grummon, W. Pratt, and R. Loloee, Mater. Res. Soc. Symp. Proc. 187, 137 (1990).

${ }^{6}$ F. T. Cheng, P. Shi, and H. C. Man, Scr. Mater. 45, 1083 (2001).

${ }^{7}$ Y. Fu, W. Huang, H. Du, X. Huang, J. Tan, and X. Gao, Surf. Coat. Technol. 145, 107 (2001).

${ }^{8}$ K. Gall, M. L. Dunn, Y. Liu, P. Labossiere, H. Sehitoglu, and Y. I. Chumlyakov, J. Eng. Mater. Technol. 124, 238 (2002).

${ }^{9}$ D. Lee, B. Park, A. Saxena, and T. Serene, J. Endod. 22, 543 (1996).

${ }^{10}$ R. Liu, D. Y. Li, Y. S. Xie, R. Llewellyn, and H. M. Hawthorne, Scr. Mater. 41, 691 (1999).

${ }^{11}$ R. Liu and D. Y. Li, Metall. Mater. Trans. A 31A, 2773 (2000).

${ }^{12}$ S. Moyne, C. Poilane, K. Kitamura, S. Miyazaki, P. Delobelle, and C. Lexcellent, Mater. Sci. Eng., A 273-275, 727 (1999).

${ }^{13}$ W. Ni, Y. Cheng, and D. Grummon, Appl. Phys. Lett. 80, 3310 (2002).

${ }^{14}$ J. S. Villarrubia, J. Res. Natl. Inst. Stand. Technol. 102, 425 (1997).

${ }^{15}$ K. L. Johnson, Contact Mechanics (Cambridge University Press, Cambridge, 1994), p. 171. 